\title{
Two-dimensional analysis of the double-resonant $2 D$ Raman mode in bilayer graphene
}

\author{
Felix Herziger, ${ }^{1, *}$ Matteo Calandra,${ }^{2}$ Paola Gava, ${ }^{2}$ Patrick May, ${ }^{1}$ \\ Michele Lazzeri, ${ }^{2}$ Francesco Mauri, ${ }^{2}$ and Janina Maultzsch ${ }^{1}$ \\ ${ }^{1}$ Institut für Festkörperphysik, Technische Universität Berlin, Hardenbergstrasse 36, 10623 Berlin, Germany \\ ${ }^{2}$ Institut de Minéralogie, de Physique des Matériaux, et de Cosmochimie, \\ UMR CNRS 7590, Sorbonne Universités - UPMC Univ Paris 06, \\ MNHN, IRD, 4 Place Jussieu, F-75005 Paris, France
}

\begin{abstract}
By computing the double-resonant Raman scattering cross-section completely from first principles and including electron-electron interaction at the $G W$ level, we unravel the dominant contributions for the double-resonant $2 D$-mode in bilayer graphene. We show that, in contrast to previous works, the so-called inner processes are dominant and that the $2 D$-mode lineshape is described by three dominant resonances around the $K$ point. We show that the splitting of the TO phonon branch in $\Gamma-K$ direction, as large as $12 \mathrm{~cm}^{-1}$ in $G W$ approximation, is of great importance for a thorough description of the $2 D$-mode lineshape. Finally, we present a method to extract the TO phonon splitting and the splitting of the electronic bands from experimental data.

PACS numbers: 78.30.-j, 78.67.Wj, 81.05.ue, 63.22.Rc
\end{abstract}

Double-resonance Raman spectroscopy provides a versatile tool for investigating the electronic structure and phonon dispersion of graphitic systems by tuning the laser energy [1]. In particular, the $D$ and $2 D$ Raman modes allow to investigate structural changes, such as the number of layers, disorder, strain and doping in the sample [2-8].

Especially the distinction between single, bi-, and fewlayer graphene via measuring the $2 D$ mode attracted great attention due to its simplicity [2]. In single-layer graphene, the double-resonance is often simplified to one single scattering process, well describing the experimental peak shape of the $2 D$ mode. Up to now, the $2 D$ mode in bilayer graphene is described and interpreted within the framework of four scattering processes. Each process was assigned to a different spectral feature in the $2 D$-mode lineshape, phenomenologically explaining the observed peakshape [2]. All successive studies on the $2 D$ mode in bilayer graphene relied on this assignment [9-14]. Furthermore, the $2 D$ mode in bilayer graphene has been mainly discussed in terms of outer processes $[2,9-11,14]$. However, the importance of inner processes was shown both theoretically and experimentally for the $2 D$ mode in single-layer graphene $[5,15,16]$. In bilayer graphene, only very few works considered the possibility of contributions from inner processes, but were still neglecting the splitting of the two TO (transversal optical) phonon branches $[12,13]$. Hence, the role of different contributions to the double-resonance in bilayer graphene is still under discussion and needs final clarification.

In this letter, by completely calculating the doubleresonant Raman cross-section from first principles and by comparing with experimental spectra for different laser energies, we unravel the dominant scattering processes in bilayer graphene. In contrast to previous works that explained the $2 D$-mode lineshape with four independent scattering processes $[2,9-14]$, we show that the $2 D$ mode is described by three dominant resonances around the $K$ point from inner processes plus a weaker contribution from outer processes. We show that the $G W$ correction to the TO phonon branch leads to a much larger TO splitting than in LDA approximation. This splitting cannot be neglected; we present an analysis to directly derive the TO phonon and electronic splitting in bilayer graphene with high accuracy.

Experimental Raman spectra were obtained from freestanding bilayer graphene in back-scattering geometry under ambient conditions using a Horiba HR800 spectrometer with a 1800 lines $/ \mathrm{mm}$ grating with spectral resolution of $1 \mathrm{~cm}^{-1}$. During all measurements the laser power was kept below $0.5 \mathrm{~mW}$ to avoid sample damaging or heating. Spectra were calibrated by standard neon lines. The freestanding bilayer graphene enables us to probe the intrinsic $2 D$-mode lineshape, ensuring an accurate extraction of the fitting parameters [16], following the model of Basko [17].

In Bernal-stacked bilayer graphene the $\pi$ orbitals give rise to two valence and two conduction bands, denoted as $\pi_{1}, \pi_{2}$ and $\pi_{1}^{*}, \pi_{2}^{*}$. Bilayer graphene possesses two TO phonon branches, each one degenerate with an LO (longitudinal optical) branch at $\Gamma$. At $\Gamma$ the TO branches are split into a symmetric and an anti-symmetric vibration. The symmetric TO phonon is an in-phase vibration between the lower and upper layer and exhibits $E_{g}$ symmetry in the point group $D_{3 d}$, whereas the anti-symmetric vibration is out-of-phase ( $E_{u}$ symmetry). Our calculated frequency splitting at $\Gamma$ is approx. $5 \mathrm{~cm}^{-1}$, comparable with the experimentally observed $6 \mathrm{~cm}^{-1}$ splitting in graphite [18]. Along the $\Gamma-K$ direction, the $G W$ calculated TO splitting increases to values as large as $12 \mathrm{~cm}^{-1}$, whereas the splitting in LDA is approximately two times smaller. The displacement patterns of the TO vibrations change away from $\Gamma$, we however extend the labeling of the phonon branches throughout the BZ. 

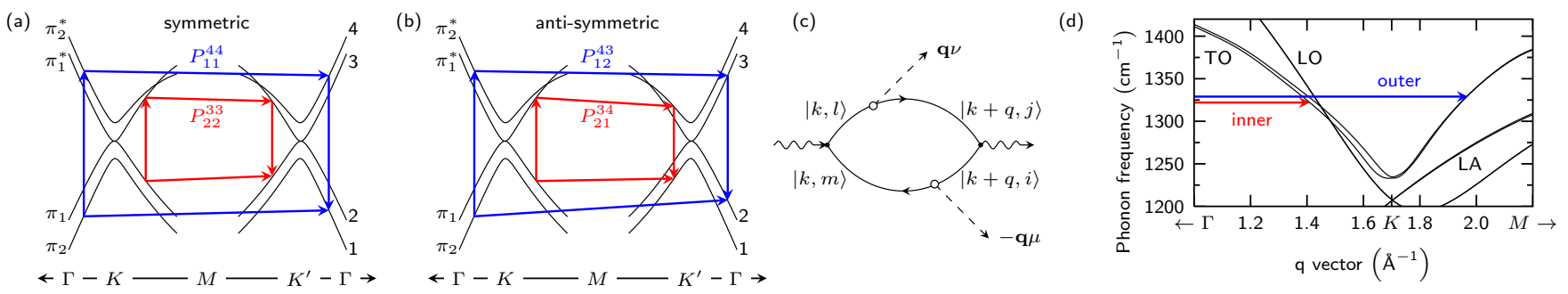

Figure 1. Schematized illustration of the $2 D$-mode scattering processes along the $\Gamma-K-M-K^{\prime}-\Gamma$ high-symmetry direction for (a) symmetric and (b) anti-symmetric processes. Inner and outer processes are marked with red and blue traces, respectively. (c) Goldstone diagram for a double-resonant $e$ - $h$ scattering process $P_{m i}^{l j}$. (d) $G W$-corrected phonon dispersion of bilayer graphene close to $K$, showing the TO splitting in $\Gamma-K$ direction.

The double-resonant $2 D$ mode is a second-order Raman process, involving two TO phonons with wave vector $\mathbf{q} \neq 0$. The process can be divided into four virtual transitions, (i) creation of an electron-hole pair by a photon with energy $\hbar \omega_{L}$, (ii) scattering of an electron/hole state by a phonon with wave vector $\mathbf{q}$, (iii) scattering of an electron/hole state by a phonon with wave vector $-\mathbf{q}$ and, (iv) recombination of the electronhole pair. The observed frequency of this process is twice the phonon frequency at $\mathbf{q}$. As we have explicitly verified, the processes where one phonon is scattered by an electron and one phonon is scattered by a hole (diagrams of the kind shown in Fig. 1), are, by far, the most dominant contribution to the Raman cross-section [15]. We will refer to these processes as electron-hole scattering (e-h scattering). The scattering processes can be further divided into symmetric/anti-symmetric and inner/outer processes. Symmetric processes are scattering events between equivalent electronic bands at $K$ and $K^{\prime}$, whereas for an anti-symmetric process the band index is changing. We refer to the term inner process, if the resonant phonon wave-vector stems from a sector of $\pm 30^{\circ}$ next to the $K-\Gamma$ direction with respect to $K$. Conversely, outer processes have phonon wave-vectors from $\pm 30^{\circ}$ next to the $K-M$ directions [Fig. 2(a)]. To simplify the labeling of the scattering processes, we enumerate the electronic bands starting from the energetically lowest band near $K$. Every scattering process $P_{m i}^{l j}$ is then uniquely defined by four indices that are given by the band indices of the initial electron $m$, of the excited electron $l$, of the scattered electron $j$, and of the scattered hole $i$. Since the incoming light couples mostly to only two $(1 \rightarrow 4$ and $2 \rightarrow 3$ ) of the four possible optical transitions, four different combinations of $e-h$ scattering are allowed [2, 19]. These are the symmetric processes $P_{11}^{44}$ and $P_{22}^{33}$ and the anti-symmetric processes $P_{12}^{43}$ and $P_{21}^{34}$.

Following Ref. [15], the two-phonon $(p p)$ doubleresonant Raman intensity is

$$
\begin{aligned}
I(\omega)= & \frac{1}{N_{q}} \sum_{\mathbf{q}, \nu, \mu} I_{\mathbf{q} \nu \mu}^{p p} \delta\left(\omega_{L}-\omega-\omega_{-\mathbf{q}}^{\nu}-\omega_{\mathbf{q}}^{\mu}\right) \\
& {\left[n\left(\omega_{-\mathbf{q}}^{\nu}\right)+1\right]\left[n\left(\omega_{\mathbf{q}}^{\mu}\right)+1\right], }
\end{aligned}
$$

where $\omega_{\mathbf{q}}^{\mu}$ and $n\left(\omega_{\mathbf{q}}^{\mu}\right)$ are the phonon frequencies and the Bose distributions for mode $\mu$, respectively [20]. The probability of exciting two phonons is $I_{\mathbf{q} \nu \mu}^{p p}=$ $\left|\frac{1}{N_{k}} \sum_{\mathbf{k}, \beta} K_{\beta}^{p p}(\mathbf{k}, \mathbf{q}, \nu, \mu)\right|^{2}$, where the matrix elements $K_{\beta}^{p p}(\mathbf{k}, \mathbf{q}, \nu, \mu)$ are defined by expressions involving the electron and phonon band dispersion, the electronphonon coupling $g_{\mathbf{k} n, \mathbf{k}+\mathbf{q} m}^{\mu}$ and the electron-light $D_{\mathbf{k} n, \mathbf{k} m}$ matrix-elements throughout the full BZ (see appendix A of Ref. [15]). Here, $\mathbf{k}$ refers to the electron wave-vector and $\beta$ labels the different possibilities of electron and hole scattering. We want to remind the reader of the importance of quantum interference in the double-resonance process. Scattering processes with the same final state $(\mathbf{q}, \mu, \nu)$ but different intermediate states can observe interference. Consequently, scattering processes at different $\mathbf{q}$ do not interfere. In most previous works on the $2 D$ mode in bilayer graphene, the interference between different processes was completely neglected. However, as will be shown later, quantum interference has remarkable impact on the $2 D$-mode lineshape in bilayer graphene.

Due to the difficulties in obtaining $g_{\mathbf{k} n, \mathbf{k}+\mathbf{q} m}^{\mu}$ and $D_{\mathbf{k} n, \mathbf{k} m}$ directly from first principles, previous publications used matrix elements derived from tight-binding models [15, 21, 22]. Here, we overcome this difficulty by using Wannier interpolation [23] of the electron-phonon and the electron-light matrix elements, as developed in Ref. [24]. We first calculate from first principles in LDA approximation [25] the unscreened electric dipole and the screened electron-phonon matrix elements on a $64 \times 64$ electron-momentum grid and a $6 \times 6$ phonon momentum grid. We then interpolate them to denser $480 \times 480$ electron-momentum grid randomly shifted from the origin and a 12288-points phonon momentum grid, covering a sufficiently large region around the $K$ points. The phonon bands were Fourier interpolated from a $12 \times 12$ phonon momentum grid. The electronic, the TO phonon bands, and $g_{\mathbf{k} n, \mathbf{k}+\mathbf{q} m}^{\mu}$ were $G W$-corrected, following the approach given in Ref. [15] (see SI [30]). The electron broadening $\gamma$ was chosen to be twice as large as that in Ref. [15] to account for additional electron-electron interaction [26], namely $\gamma=0.081832 \times\left(\hbar \omega_{L} / 2-0.1645\right)$ 

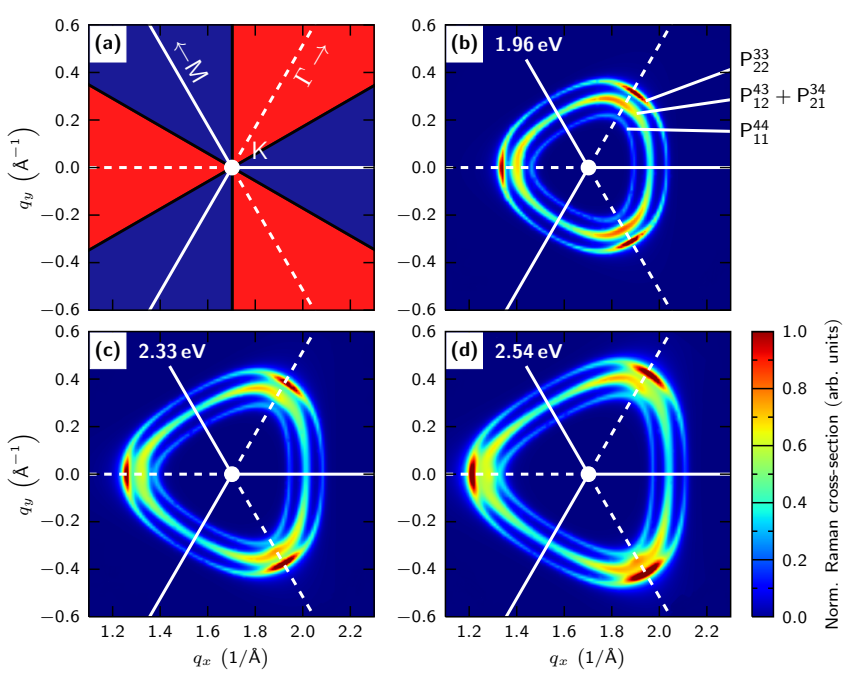

Figure 2. (a) Illustration of the phonon wave-vector sectors for inner (red) and outer (blue) processes around the $K$ point. The solid and dashed white lines denote the $K-M$ and $K-\Gamma$ high-symmetry lines, respectively. (b)-(d) Plots of the normalized $2 D$-mode scattering cross-section $\mathcal{I}_{\mathbf{q}}$ around the $K$ point for different laser energies.

$\mathrm{eV}$. This choice gives better agreement with experiments (SI). Finally, the $\delta$-function in Eq. (1) is broadened with an $8 \mathrm{~cm}^{-1}$ Lorentzian [27].

Fig. 2 presents calculated contour plots of $\mathcal{I}_{\mathbf{q}}=$ $\sum_{\nu, \mu} I_{\mathbf{q} \nu \mu}^{p p}$ for the double-resonant $2 D$ mode in bilayer graphene for various $\hbar \omega_{L}$. Three resonances around the $K$ point contribute to the $2 D$ mode. These regions are attributed to, from inside to outside, the $P_{11}^{44}$, the antisymmetric $P_{12}^{43}$ and $P_{21}^{34}$, and the $P_{22}^{33}$ processes. As the resonant phonon wave-vectors of the anti-symmetric processes are nearly degenerated, the resulting phonon frequencies are very similar, disproving previous assignments of anti-symmetric processes to different spectral features of the $2 D$ mode $[2,9-14]$. Furthermore, the dominant contributions to the $2 D$-mode scattering crosssection stem from the $K-\Gamma$ direction which can be identified with inner processes.

We will now turn our discussion to the calculated Raman spectra of the $2 D$ mode in bilayer graphene. Fig. 3 compares the calculated Raman spectra with spectra from freestanding bilayer graphene at different $\hbar \omega_{L}$. The overall agreement between calculation and experimental data is very good, although there is a slight mismatch in frequency. The calculated frequencies are approximately $10 \mathrm{~cm}^{-1}$ too high, yet our calculations reproduce the lineshape of the $2 D$ mode, i.e., the relative intensities of the different contributions, very well.

Fig. 4 shows the decomposition of the calculated $2 D$ mode spectrum at $1.96 \mathrm{eV}$ excitation energy into its different contributions. The decomposition for other $\hbar \omega_{L}$ is accordingly (SI). As in single-layer graphene, we confirm that in bilayer graphene the $e-h$ scattering processes dom-

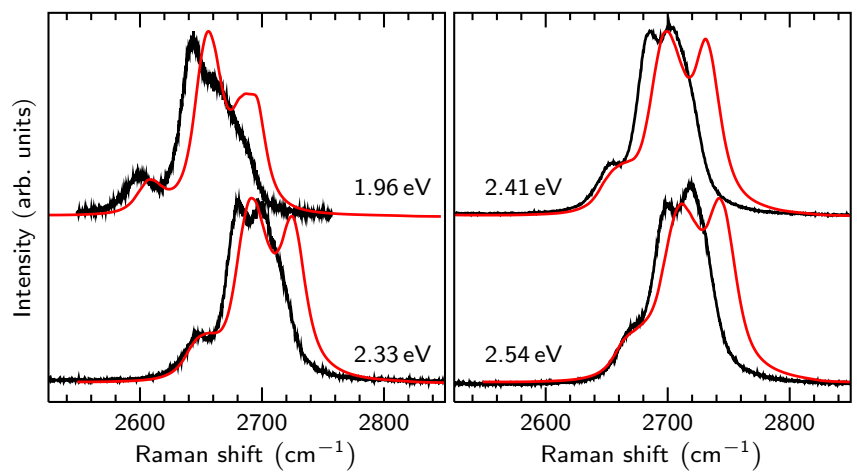

Figure 3. Comparison of calculated $2 D$-mode spectra with Raman spectra from freestanding bilayer graphene at different $\hbar \omega_{L}$. Calculations and experimental data are shown as red and black curves, respectively. Spectra are normalized and vertically offset.

inate compared to all other scattering paths. Furthermore, inner processes dominate over outer ones. By explicitly decomposing the $2 D$ mode into the four different processes in Fig. 4(b), we find that the symmetric $P_{11}^{44}$ and $P_{22}^{33}$ processes are on the low- and high-frequency side of the $2 D$ mode, respectively. The frequencies of the antisymmetric processes are in between the symmetric contributions and nearly degenerate, as already inferred from Fig. 2. This disagrees with all previous works [2, 9-14], attributing substantially different phonon frequencies to the anti-symmetric processes. As seen in Fig. 4(b), the decomposition of the single processes is not additive, i.e., the sum of the four processes does not yield the total spectrum. This can be directly attributed to quantum interference effects between the anti-symmetric processes. By decomposing the spectrum into the single processes as in Fig. 4(b), interference between the $P_{m i}^{l j}$ is prohibited. However, $P_{12}^{43}$ and $P_{21}^{34}$ exhibit a large overlap in reciprocal space and interfere constructively. Decomposing the total spectrum into symmetric and anti-symmetric contributions and thus allowing interference between those processes yields the spectrum in Fig. 4(c). This decomposition is additive. The constructive interference has remarkable impact on the $2 D$-mode lineshape, i.e., the intensity of the anti-symmetric processes is drastically enhanced, highlighting the importance of quantum interference effects in the double-resonance process.

Up to now, we described the $2 D$ mode in terms of three dominant resonances that split up into inner and outer contributions. Thus, one might expect six separate peaks in the $2 D$-mode spectrum in total. This is in contrast to the experimentally observed lineshape, where usually three or four peaks can be distinguished. However, the decomposition in Fig. 4(a) and (b) shows that inner and outer contributions for the $P_{11}^{44}, P_{12}^{43}$, and $P_{21}^{34}$ processes are nearly degenerate in frequency, thus reducing the number of observable $2 D$-mode peaks for these 

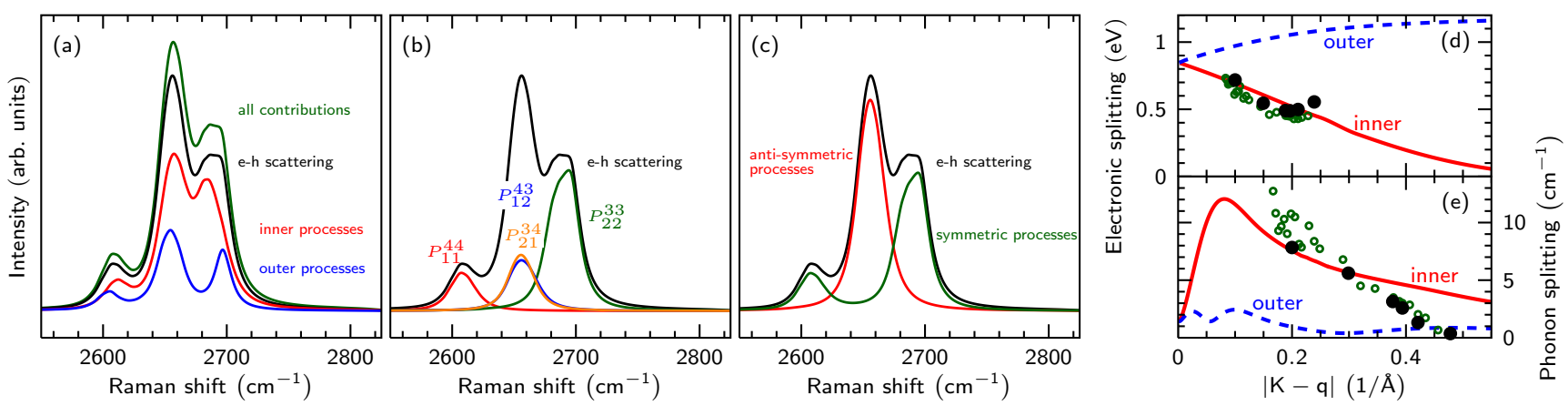

Figure 4. Calculated 2D-mode spectra at $1.96 \mathrm{eV}$ excitation energy. Decomposition of the calculated spectra into (a) $e$ - $h$ scattering processes, as well as inner and outer contributions, (b) the four different scattering processes $P_{m i}^{l j}$ (without interference between the different processes), and (c) into symmetric and anti-symmetric processes (including interference). (d) and (e) show the experimental values for the electronic and TO phonon splitting, respectively. The solid (red) and dashed (blue) lines denote the DFT calculated splittings in inner and outer direction, respectively. Open, green circles are data points from Ref. [11]. Filled, black circles represent data points from this work.

processes to two. Only the $P_{22}^{33}$ process exhibits a splitting between inner and outer contributions that is large enough to be detected in experiments; it is responsible for the third and fourth peak in the $2 D$-mode lineshape. Therefore, in experiments the $2 D$ mode should be fitted with four peaks, where the assignment of the peaks, from lowest to highest frequency, is $P_{11}^{44}, P_{12}^{43} / P_{21}^{34}$, inner $P_{22}^{33}$, and outer $P_{22}^{33}$. In previous works, the inner $P_{22}^{33}$ contribution was erroneously assigned to an anti-symmetric scattering process, whereas the outer contribution, i.e., the small high-frequency shoulder of the $2 D$ mode, was attributed to a symmetric process. Here, we showed that these two peaks result from the same scattering process $\left(P_{22}^{33}\right)$. Our assignment of the third and fourth $2 D$-mode peaks to inner and outer $P_{22}^{33}$ contributions is supported by recent experiments on strained bilayer graphene [28]. Due to different dispersions of inner and outer processes, both contributions to $P_{22}^{33}$ merge with increasing laser energy. Therefore, at higher laser energies the fourth peak vanishes. This can be seen in the spectrum of the freestanding bilayer graphene at $2.54 \mathrm{eV}$ excitation energy in Fig. 3. Here, the small high-frequency shoulder cannot be identified any more, giving further evidence to our assignment of the three dominant contributions to the $2 D$ mode in bilayer graphene.

In previous works, the TO splitting in bilayer graphene has always been neglected in the double-resonance, as only outer processes were considered and the TO splitting along $K-M$ is of the order of $1.5 \mathrm{~cm}^{-1}$ [2]. However, we proved that inner processes are dominant. In fact, along $K-\Gamma$ the TO splitting is as large as $12 \mathrm{~cm}^{-1}$ in $G W$ approximation. We observe that the dominant contributions to symmetric processes stem from scattering with symmetric TO phonons, whereas the dominant contributions to anti-symmetric processes result from scattering with anti-symmetric TO phonons (see SI).

The fact that symmetric and anti-symmetric processes couple to different phonon branches has remarkable impact on the $2 D$-mode lineshape. If all scattering processes would couple to the same phonon branch, all contributions would be equidistantly spaced in frequency. This is true for outer contributions [Fig. 4(a)], since the TO splitting along $K-M$ is neglibile. However, the dominant contributions stem from inner processes and therefore, the TO splitting must be taken into account. Since the inner anti-symmetric processes couple to the energetically higher TO branch along $K-\Gamma$, their frequency is upshifted with respect to the center between the symmetric processes. This upshift is a direct measure of the TO splitting and can be easily accessed experimentally. Furthermore, one can also extract the splitting of the electronic bands from the $2 D$-mode spectrum, as this parameter is directly connected to the frequency difference between the symmetric processes and the laser-energy dependent shift rate of the $2 D$ mode (see SI). Figures $4(\mathrm{~d}$ ) and (e) present the measured TO phonon and electronic splitting in comparison with data from DFT $+G W$ calculations. As can be seen, the experimental values are in good agreement with the calculated curve along the inner direction. However, a discrepancy in the TO splitting between theory and experiment can be observed for q vectors close to $K$. The TO phonon splitting is largest along $\Gamma-K$ and decreases away from this high-symmetry line. Since also phonons away from the high-symmetry direction contribute to the double resonance [29], the experimentally measured TO splitting is expected to be smaller than theoretically predicted along $\Gamma-K$. Thus, the theoretical curves should represent lower and upper limits for the experimental values. The fact that the experimental data is also outside those boundaries indicates that the commonly assumed $G W$ correction might still underestimate the TO splitting, which is probably larger than $15 \mathrm{~cm}^{-1}$ close to $K$. Finally, we should note that all results for the $2 D$ mode in bilayer graphene are also 
valid for the $D$ mode.

In conclusion, we demonstrated that the doubleresonant $2 D$ Raman mode in bilayer graphene is described by three dominant contributions, contradicting all previous works on this topic. We showed that inner processes contribute most to the Raman scattering cross-section, as in single-layer graphene. Moreover, we demonstrated that the TO phonon splitting is of great importance for a correct analysis of the $2 D$-mode lineshape. The TO phonon and electronic splitting can be directly extracted from experimental Raman spectra using the presented analysis. Our results highlight the key role of inner processes and finally clarify the origin of the complex $2 D$-mode lineshape in bilayer graphene.

FH, PM, and JM acknowledge financial support from the DFG under Grant no. MA 4079/3-1 and the European Research Council (ERC) Grant no. 259286. MC and FM acknowledge support from the Graphene Flagship and from the French state funds (reference ANR-11IDEX-0004-02, ANR-11-BS04-0019 and ANR-13-IS100003-01). Computer facilities were provided by CINES, CCRT and IDRIS (project no. x2014091202).

*fhz@physik.tu-berlin.de

[1] C. Thomsen and S. Reich, Phys. Rev. Lett. 85, 5214 (2000).

[2] A. C. Ferrari, J. C. Meyer, V. Scardaci, C. Casiraghi, M. Lazzeri, F. Mauri, S. Piscanec, D. Jiang, K. S. Novoselov, S. Roth, and A. K. Geim, Phys. Rev. Lett. 97, 187401 (2006).

[3] S. Berciaud, S. Ryu, L. E. Brus, and T. F. Heinz, Nano Letters 9, 346 (2009).

[4] T. M. G. Mohiuddin, A. Lombardo, R. R. Nair, A. Bonetti, G. Savini, R. Jalil, N. Bonini, D. M. Basko, C. Galiotis, N. Marzari, K. S. Novoselov, A. K. Geim, and A. C. Ferrari, Phys. Rev. B 79, 205433 (2009).

[5] M. Mohr, J. Maultzsch, and C. Thomsen, Phys. Rev. B 82, 201409 (2010).

[6] M. Huang, H. Yan, C. Chen, D. Song, T. Heinz, and J. Hone, PNAS 106, 7304 (2009).

[7] M. Huang, H. Yan, T. F. Heinz, and J. Hone, Nano Lett. 10, 4074 (2010).

[8] O. Frank, M. Mohr, J. Maultzsch, C. Thomsen, I. Riaz, R. Jalil, K. Novoselov, G. Tsoukleri, J. Parthenios, and K. Papagelis, ACS Nano 5, 2231 (2011).

[9] L. M. Malard, J. Nilsson, D. C. Elias, J. C. Brant, F. Plentz, E. S. Alves, A. H. Castro Neto, and M. A. Pimenta, Phys. Rev. B 76, 201401 (2007).

[10] L. G. Cançado, A. Reina, J. Kong, and M. S. Dresselhaus, Phys. Rev. B 77, 245408 (2008).

[11] D. L. Mafra, L. M. Malard, S. K. Doorn, H. Htoon, J. Nilsson, A. H. Castro Neto, and M. A. Pimenta, Phys. Rev. B 80, 241414 (2009).

[12] D. Mafra, E. Moujaes, S. Doorn, H. Htoon, R. Nunes, and M. Pimenta, Carbon 49, 1511 (2011).

[13] D. L. Mafra, J. Kong, K. Sato, R. Saito, M. S. Dresselhaus, and P. T. Araujo, Nano Lett. 12, 2883 (2012).
[14] H. Wang, J. You, L. Wang, M. Feng, and Y. Wang, J. Raman Spectrosc. 41, 125 (2010).

[15] P. Venezuela, M. Lazzeri, and F. Mauri, Phys. Rev. B 84, 035433 (2011).

[16] S. Berciaud, X. Li, H. Htoon, L. E. Brus, S. K. Doorn, and T. F. Heinz, Nano Letters 13, 3517 (2013).

[17] D. M. Basko, Phys. Rev. B 78, 125418 (2008).

[18] P. Eklund, J. Holden, and R. Jishi, Carbon 33, 959 (1995).

[19] L. M. Malard, M. H. D. Guimarães, D. L. Mafra, M. S. C. Mazzoni, and A. Jorio, Phys. Rev. B 79, 125426 (2009).

[20] We discuss here the scattering in terms of "doubleresonance", since the dominant phonon momentum is determined by the two resonant conditions on the light absorption and emission (see, e.g., Sec. III E.2 and Fig. 23 of Ref. [15]). However, triple-resonant processes also occur and are fully included in our calculations.

[21] R. Narula, N. Bonini, N. Marzari, and S. Reich, phys. stat. sol. (b) 248, 2635 (2011).

[22] R. Narula, N. Bonini, N. Marzari, and S. Reich, Phys. Rev. B 85, 115451 (2012).

[23] A. A. Mostofi, J. R. Yates, Y.-S. Lee, I. Souza, D. Vanderbilt, and N. Marzari, Comput. Phys. Commun. 178, 685 (2008).

[24] M. Calandra, G. Profeta, and F. Mauri, Phys. Rev. B 82, 165111 (2010).

[25] P. Giannozzi, S. Baroni, N. Bonini, M. Calandra, R. Car, C. Cavazzoni, D. Ceresoli, G. L. Chiarotti, M. Cococcioni, I. Dabo, A. D. Corso, S. de Gironcoli, S. Fabris, G. Fratesi, R. Gebauer, U. Gerstmann, C. Gougoussis, A. Kokalj, M. Lazzeri, L. Martin-Samos, N. Marzari, F. Mauri, R. Mazzarello, S. Paolini, A. Pasquarello, L. Paulatto, C. Sbraccia, S. Scandolo, G. Sclauzero, A. P. Seitsonen, A. Smogunov, P. Umari, and R. M. Wentzcovitch, J. Phys.: Condens. Matter 21, 395502 (2009).

[26] K. F. Mak, F. H. da Jornada, K. He, J. Deslippe, N. Petrone, J. Hone, J. Shan, S. G. Louie, and T. F. Heinz, Phys. Rev. Lett. 112, 207401 (2014).

[27] L. Paulatto, F. Mauri, and M. Lazzeri, Phys. Rev. B 87, 214303 (2013).

[28] O. Frank, M. Bouša, I. Riaz, R. Jalil, K. S. Novoselov, G. Tsoukleri, J. Parthenios, L. Kavan, K. Papagelis, and C. Galiotis, Nano Letters 12, 687 (2012).

[29] P. May, M. Lazzeri, P. Venezuela, F. Herziger, G. Callsen, J. S. Reparaz, A. Hoffmann, F. Mauri, and J. Maultzsch, Phys. Rev. B 87, 075402 (2013).

[30] See Supplemental Material, which includes Refs. 11, 15, $17,24,26,31-34$.

[31] M. Lazzeri, C. Attaccalite, L. Wirtz, and F. Mauri, Phys. Rev. B 78, 081406 (2008).

[32] A. Grüneis, J. Serrano, A. Bosak, M. Lazzeri, S. L. Molodtsov, L. Wirtz, C. Attaccalite, M. Krisch, A. Rubio, F. Mauri, and T. Pichler, Phys. Rev. B 80, 085423 (2009).

[33] J. A. Yan, W. Y. Ruan, and M. Y. Chou, Phys. Rev. B 77, 125401 (2008).

[34] F. Kadi and E. Malic, Phys. Rev. B 89, 045419 (2014). 


\title{
Supplementary material for the paper:
}

\section{Two-dimensional analysis of the double-resonant $2 D$ Raman mode in bilayer graphene}

\author{
Felix Herziger, ${ }^{1, *}$ Matteo Calandra, ${ }^{2}$ Paola Gava, ${ }^{2}$ Patrick May, ${ }^{1}$ \\ Michele Lazzeri, ${ }^{2}$ Francesco Mauri, ${ }^{2}$ and Janina Maultzsch ${ }^{1}$ \\ ${ }^{1}$ Institut für Festkörperphysik, Technische Universität Berlin, Hardenbergstrasse 36, 10623 Berlin, Germany \\ ${ }^{2}$ IMPMC, Université Pierre et Marie Curie, CNRS, 4 place Jussieu, 75252 Paris, France
}

\section{$G W$ correction to the TO phonon modes in bilayer graphene}

Phonon dispersions are obtained from ab initio DFT calculations in the linear-response scheme and corrected with $G W$, similar to what was done in Refs. [1-3] for single-layer graphene. In more detail, we correct the dispersion of the two TO branches near $\mathbf{K}$, namely we define

$$
\left(\omega_{\mathbf{q} \nu}^{G W}\right)^{2}=0.5 \times \operatorname{erfc}\left(\frac{\left|\mathbf{q}-\mathbf{K}_{\alpha}\right| a_{0} / 2 \pi-0.2}{0.05}\right) \times\left[\left(\alpha_{\mathbf{K}}-1\right)\left(\omega_{\mathbf{q} \nu}^{D F T}\right)^{2}+\Delta\right]
$$

where $\operatorname{erfc}()$ is the error function, $\nu=1,2$ is a label for the two TO branches, $a_{0}$ is the graphene lattice constant, and $\mathbf{K}_{\alpha}$ is the closest vector to $\mathbf{q}$ among those equivalent to $\mathbf{K}$. The constants $\alpha_{\mathbf{K}}$ and $\Delta$ are defined as $\alpha_{\mathbf{K}}=1.61$ and $\Delta=42.195 \mathrm{Ryd}^{2}$ and are identical for both TO phonon modes.

In order to apply the $G W$ correction to all phonon wave vectors in the Brillouin Zone, we use the phonon frequency Wannier interpolation method developed in Ref. [4] section III D. The comparison between the DFT and DFT $+G W$ phonon branches is shown in Fig. 1. In previous calculations at the $G W$ level, the splitting of the two TO branches along $\Gamma-K$ was found to be negligible and similar to that along $K-M$ [5], in contradiction to our results. This discrepancy is explained by the coarse $6 \times 6$ phonon-momentum mesh used in Ref. [5] and the use of Fourier interpolation. In our case, this error is not present due to the use (i) of a larger phonon momentum grid $(12 \times 12)$ and (ii) of a Wannier interpolation scheme to obtain phonon frequencies at any electron and phonon momentum in the Brillouin zone with high accuracy [4].
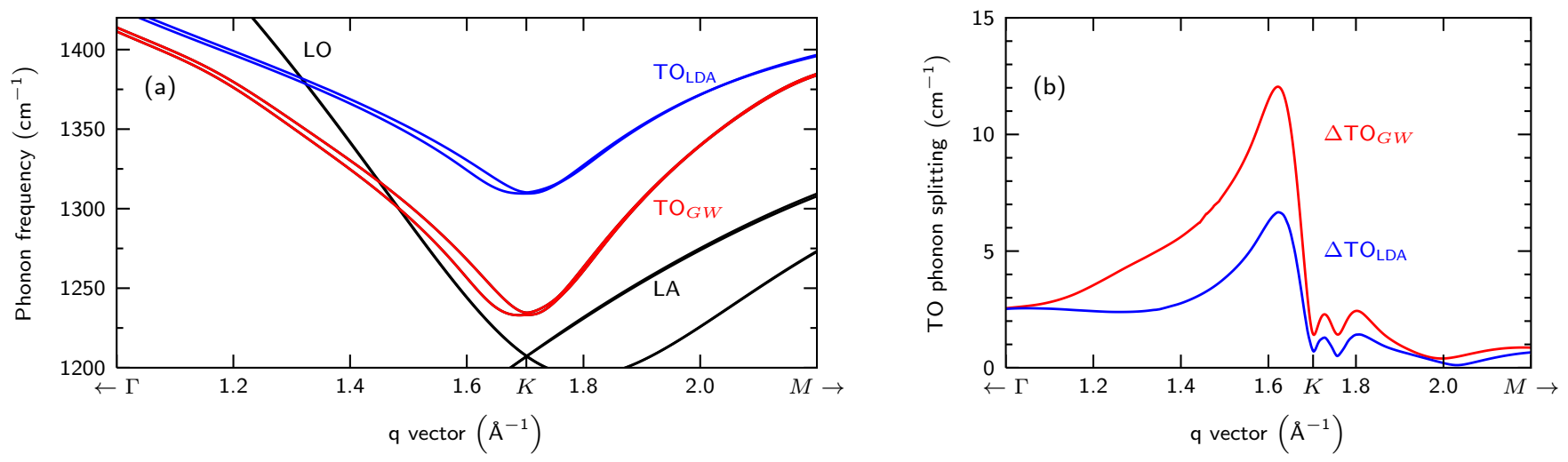

Figure 1. (a) Comparison of TO phonon frequencies in bilayer graphene calculated in LDA approximation (blue curve) and with $G W$ correction (red curve). LO and LA phonon branches were not $G W$ corrected. The numbers along the LO and TO branches refer to their branch indices at this specific wave vector. (b) Splitting of the two TO phonon branches along the $\Gamma-K-M$ high-symmetry direction in bilayer graphene. Colors are chosen as in (a). 


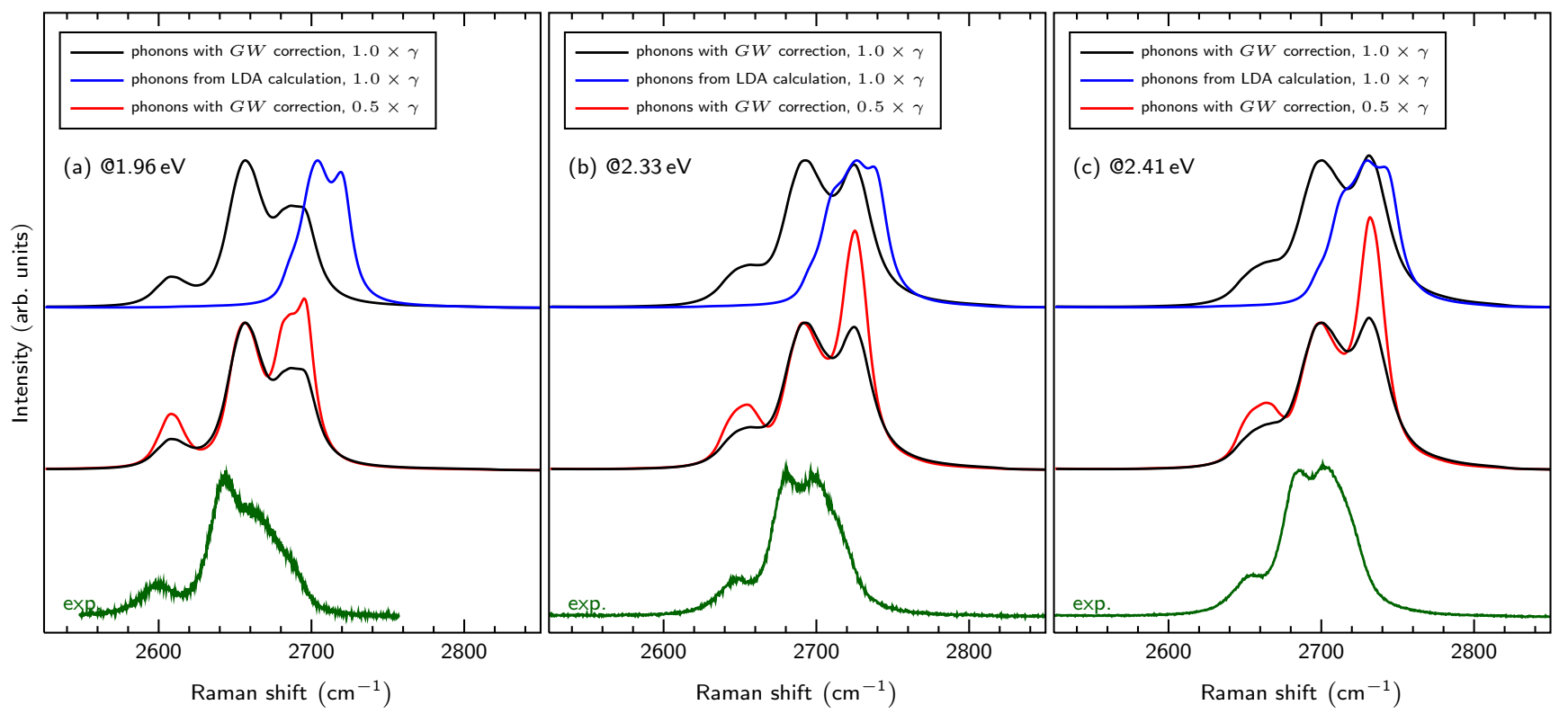

Figure 2. Calculated Raman spectra of the $2 D$ mode for different broadenings $\gamma$ and with/without $G W$ correction for the TO phonons in comparison with experimental spectra from freestanding bilayer graphene.

\section{Influence of electronic broadening and $G W$ correction on the calculated Raman spectra}

The electronic broadening $\gamma$ in our calculations was calculated according to

$$
\gamma=0.081832 \times\left(\frac{\hbar \omega_{L}}{2}-0.1645\right) \mathrm{eV}
$$

where $\hbar \omega_{L}$ represents the laser excitation energy. Using this formula, we found very good agreement between the calculated $2 D$-mode spectra and the experimentally observed lineshape. Using Eq. (2) for calculation of the electronic broadening yields values twice as large as in Ref. [3]. However, Ref. [3] only considered electron-phonon interactions for the derivation of the broadening and neglected electron-electron interaction. However, it was shown recently by Mak et al. that electron-electron interaction can contribute as much as electron-phonon interactions to the decay rate of excited charge carriers [6]. In fact, the decay rates nearly double by including electron-electron interaction. Another recent work on the optical properties of bilayer graphene used electronic broadenings as large as $125 \mathrm{meV}$, but argued that the real value must be even larger to reproduce the broad excitonic $M$-point absorption peak [7].

Figure 2 compares experimental and theoretical spectra calculated with different electronic broadenings $\gamma$ for different laser excitation energies. For comparison, the spectra are normalized to the contribution of the anti-symmetric processes. As can be seen, the spectra calculated with electronic broadenings $0.5 \times \gamma$ do not reproduce the experimentally observed lineshape. In contrast, spectra that were calculated using electronic broadening from Eq. (2) show good agreement with experimentally obtained spectra.

Figure 2 also shows the influence of the $G W$ correction on the calculated spectra. As already inferred in Fig. 1, the calculated Raman spectra, where DFT-calculated phonon frequencies were used, exhibit frequencies that are clearly too high. Furthermore, the overall linewidth of the calculated spectra does not fit the experimentally observed lineshape, i.e., the calculated lineshape is too narrow (the same phonon broadening of $8 \mathrm{~cm}^{-1}$ was used for all calculations). In contrast to the DFT $+G W$ calculated phonon dispersion, the TO phonon branch exhibits a reduced slope in LDA approximation. Thus, the frequency separation of the different scattering processes in LDA approximation is smaller and, therefore, the linewidth is reduced. Spectra, where the DFT $+G W$ calculated TO frequencies were used, show good agreement in both the absolute frequencies and the overall linewidth of the $2 D$ mode with measured spectra of freestanding bilayer graphene. 

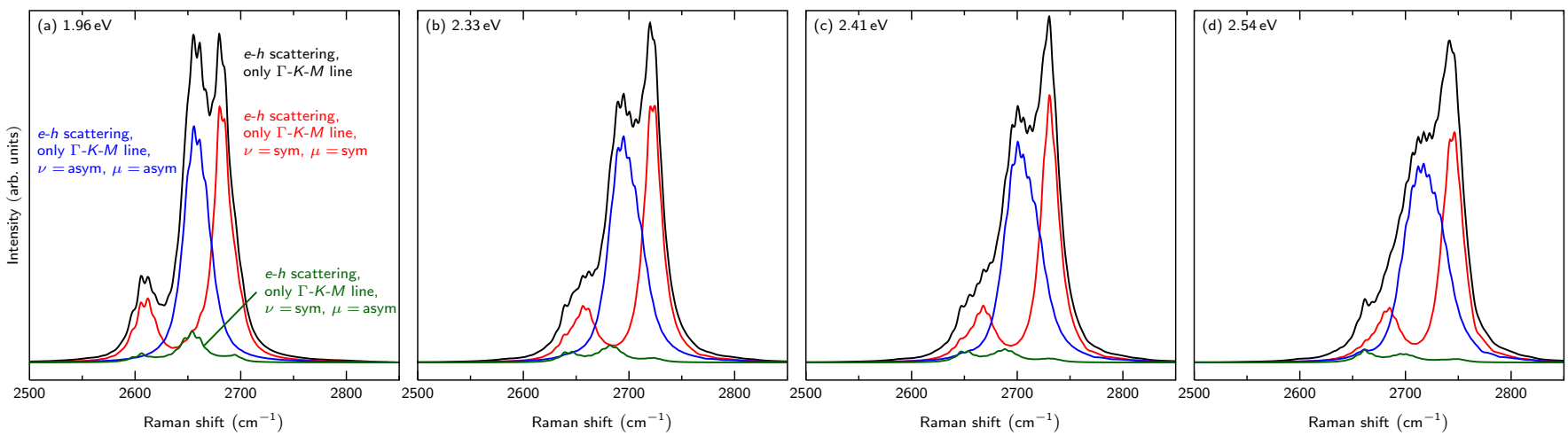

Figure 3. Calculated spectra along the $\Gamma-K-M$ high-symmetry direction. The black curve represents the calculated spectra without any restrictions on the phonon indices in Eq. (3). The contributions from scattering with symmetric phonons $(\nu=$ sym, $\mu=\mathrm{sym})$ is shown in red, contributions from scattering with anti-symmetric phonons $(\nu=$ asym, $\mu=$ asym) is shown as a blue curve. Contributions to the $2 D$ mode that result from scattering with both symmetric and anti-symmetric phonons are depicted with a green curve.

\section{Contributions to the $2 D$ mode from scattering with different TO phonons}

The coupling of different scattering processes to different TO branches in the $2 D$-mode double-resonance process of bilayer graphene has never been considered so far. In order to separate the different contributions from the two TO phonon branches to the $2 D$-mode Raman scattering cross-section we proceeded as follows. We calculated the overlap between the eigenvectors of the six highest phonon branches with the symmetric and anti-symmetric TO vibration at a $\mathbf{q}$ vector along the $K-\Gamma$ direction very close to $K$. Since outside the high-symmetry directions the symmetric and anti-symmetric TO branches cannot be separated univocally by their vibration pattern, we only considered the TO branches along the $\Gamma-K-M$ high-symmetry direction in this analysis. Next, we restricted the summation of the two-phonon double-resonant Raman intensity [3]

$$
I(\omega)=\frac{1}{N_{q}} \sum_{\mathbf{q}, \nu, \mu} I_{\mathbf{q} \nu \mu}^{p p} \delta\left(\omega_{L}-\omega-\omega_{-\mathbf{q}}^{\nu}-\omega_{\mathbf{q}}^{\mu}\right)\left[n\left(\omega_{-\mathbf{q}}^{\nu}\right)+1\right]\left[n\left(\omega_{\mathbf{q}}^{\mu}\right)+1\right]
$$

to the indices $\nu, \mu$ of the symmetric (sym) or anti-symmetric (asym) TO branches along $\Gamma-K-M$. The calculated spectra for this separation are shown in Fig. 3 for different laser excitation energies. As can be seen, the dominant contribution to the symmetric processes stems from scattering with symmetric TO phonons. Vice versa, the dominant contribution to the anti-symmetric processes stems from scattering with anti-symmetric TO phonons. Scattering with the combination of symmetric and anti-symmetric TO phonons gives only minor contribution to the $2 D$-mode intensity.

\section{Calculation of the TO phonon and electronic splitting}

The TO phonon and electronic splitting in bilayer graphene can be easily obtained from the measured $2 D$-mode spectra. First, we want to recall the assignment of the observed $2 D$-mode peaks, from lowest to highest frequency, to the different scattering processes, which is as follows:

peak $1 \rightarrow P_{11}^{44}$ (sym.), peak $2 \rightarrow P_{12}^{43} / P_{21}^{34}$ (anti-sym.), peak $3 \rightarrow$ inner $P_{22}^{33}$ (sym.), peak $4 \rightarrow$ outer $P_{22}^{33}$ (sym.)

As discussed in the main text of this letter, the $P_{22}^{33}$ peak splits up into an innner and outer contribution, which is clearly observable for smaller laser excitation energies. For the calculation of the TO phonon and electronic splitting we used the inner component, i.e., the third $2 D$-mode peak, as we want to investigate the TO splitting along $K-\Gamma$ and the electronic splitting along the $K-M$ direction. Figure 4(a) shows an exemplary fit of a measured $2 D$-mode spectrum at $2.33 \mathrm{eV}$ laser energy using a fit of the form

$$
f(\omega)=\sum_{i=1}^{4} a_{i} \cdot f_{i}\left(\omega, \omega_{i}, \Gamma_{i}\right),
$$



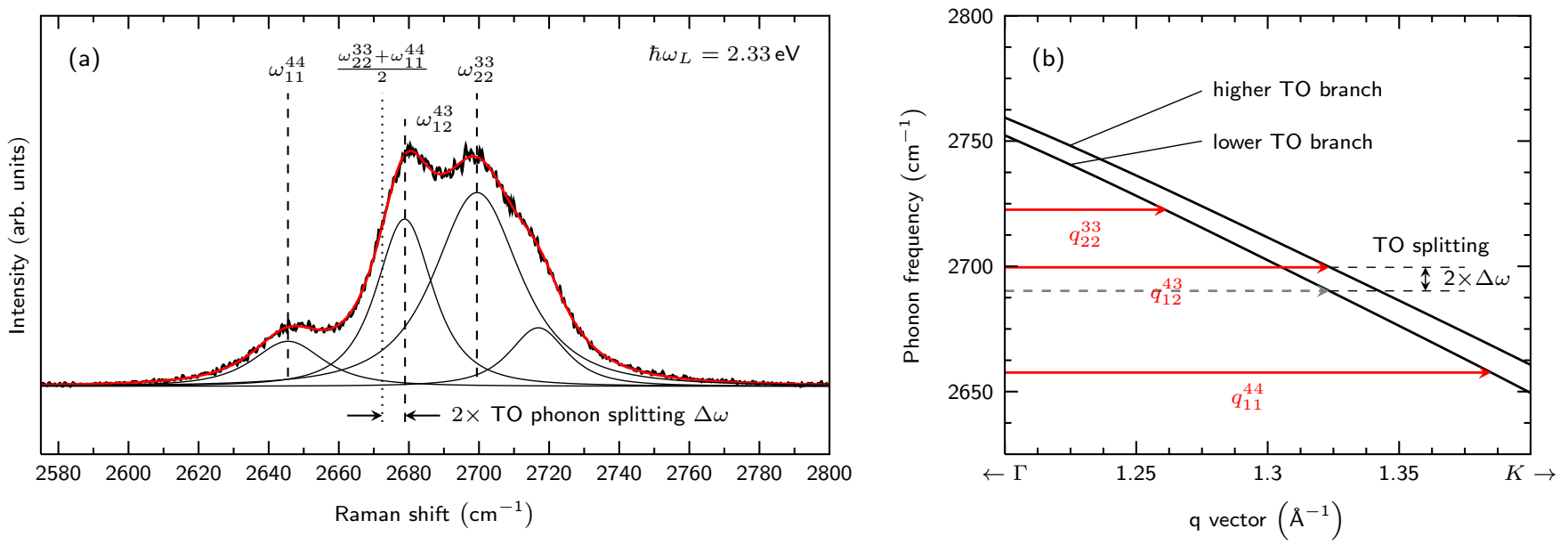

Figure 4. (a) Exemplary fit of a experimental $2 D$-mode spectrum from freestanding bilayer graphene at $2.33 \mathrm{eV}$ laser excitation energy with four peaks based on the model proposed by Basko [8]. The solid red curve represents the fit to the experimental spectrum. The peak positions of the single peaks are marked with dashed lines, the center between the symmetric scattering processes is shown by the dotted line. The upshift of the anti-symmetric processes with respect to the center between the symmetric contributions is indicated. (b) Resonant phonon wave-vectors in the phonon dispersion of bilayer graphene for $2.33 \mathrm{eV}$ laser excitation energy. The dashed, gray arrow indicates the phonon frequency of the $P_{12}^{43}$ process, if all processes would couple to the same phonon branch. The upshift of the anti-symmetric processes due to coupling to the energetically higher TO branch is indicated.

where

$$
f_{i}\left(\omega, \omega_{i}, \Gamma_{i}\right)=\left(\frac{\Gamma_{i}^{2}}{4\left(-1+2^{2 / 3}\right)\left(\omega-\omega_{i}\right)^{2}+\Gamma_{i}^{2}}\right)^{3 / 2}
$$

is a normalized function following the model of Basko [8]. Here, $a_{i}$ reflects the peak amplitude, $\omega_{i}$ represents the central peak frequency and $\Gamma_{i}$ is the full width at half maximum (FWHM). Fitting parameters for different laser excitation energies are given in Tab. I. In Fig. 4(a) the peak positions of the $P_{11}^{44}, P_{12}^{43}$, and inner $P_{22}^{33}$ processes are marked by vertical dashed lines. Furthermore, the center between the symmetric processes $P_{11}^{44}$ and inner $P_{22}^{33}$ is indicated by a dotted line. Since the main contribution to the anti-symmetric scattering processes stems from the energetically higher TO phonon branch along $\Gamma-K$, their frequency $\omega_{12}^{43}$ is upshifted with respect to the center between the symmetric processes $1 / 2 \times\left(\omega_{11}^{44}+\omega_{22}^{33}\right)$. This upshift is directly related to the TO phonon splitting. In fact, the observed upshift is two times the TO splitting, since the $2 D$ mode is a two-phonon process. Fig. 4(b) shows the situation in a different way. Here, the resonant phonon wave-vectors for the double-resonance process in bilayer graphene with $2.33 \mathrm{eV}$ laser energy are shown. The dashed, gray arrow indicates the phonon frequency of the anti-symmetric process, if this process would result from scattering with symmetric TO phonons. As we already know, the main contribution stems from scattering with anti-symmetric TO phonons and therefore, the frequency of these processes is upshifted. The effect can be easily seen in this figure. For the calculation of the electronic splitting, the frequency difference between the symmetric processes is divided by the dispersion of the $2 D$-mode peaks with laser excitation energy. Thus, the TO phonon and electronic splitting can be calculated using the following formulas

$$
\Delta \omega=\frac{1}{2}\left(\omega_{12}^{43}-\frac{\omega_{11}^{44}+\omega_{22}^{33}}{2}\right) \text { and } \Delta \varepsilon=\frac{\omega_{22}^{33}-\omega_{11}^{44}}{\mathrm{~d} \omega / \mathrm{d} \hbar \omega_{L}}
$$

where $\omega_{m i}^{l j}$ refers to the peak positions of processes $P_{m i}^{l j}$ and $\mathrm{d} \omega / \mathrm{d} \hbar \omega_{L}$ is the laser-energy dependent shift rate of the $2 D$-mode peaks. It is important to notice that the $2 D$-mode dispersion is non-linear, as can be seen from Ref. [9]. We used a quadratic fit to the data points to obtain the dispersion.

\section{Decomposition of the $2 D$ mode spectra for different laser energies}

Figure 5 presents the decomposition of the $2 D$ mode into the different contributions at different laser excitation 
Table I. Fitting parameters of experimentally obtained and theoretically calculated $2 D$-mode spectra in bilayer graphene for different laser excitation energies. For laser energies above $1.96 \mathrm{eV}$, the calculated spectra are fitted with three peaks because the fourth peak cannot be resolved any more.

\begin{tabular}{|c|c|c|c|c|c|c|c|c|c|c|c|c|}
\hline \multicolumn{13}{|c|}{ experiment } \\
\hline $\begin{array}{l}\omega_{L} \\
{[\mathrm{eV}]}\end{array}$ & $\begin{array}{c}\omega_{1} \\
{\left[\mathrm{~cm}^{-1}\right]}\end{array}$ & {$\left[\begin{array}{c}\Gamma_{1} \\
{\left[\mathrm{~cm}^{-1}\right]}\end{array}\right.$} & $\begin{array}{c}a_{1} \\
\text { [arb. u.] }\end{array}$ & $\begin{array}{c}\omega_{2} \\
{\left[\mathrm{~cm}^{-1}\right]}\end{array}$ & $\begin{array}{c}\Gamma_{2} \\
{\left[\mathrm{~cm}^{-1}\right]}\end{array}$ & $\begin{array}{c}a_{2} \\
\text { [arb. u.] }\end{array}$ & $\begin{array}{c}\omega_{3} \\
{\left[\mathrm{~cm}^{-1}\right]}\end{array}$ & $\begin{array}{c}\Gamma_{3} \\
{\left[\mathrm{~cm}^{-1}\right]}\end{array}$ & $\begin{array}{c}a_{3} \\
\text { [arb. u.] }\end{array}$ & $\begin{array}{c}\omega_{4} \\
{\left[\mathrm{~cm}^{-1}\right]}\end{array}$ & $\begin{array}{c}\Gamma_{4} \\
{\left[\mathrm{~cm}^{-1}\right]}\end{array}$ & $\begin{array}{c}a_{4} \\
\text { [arb. u.] }\end{array}$ \\
\hline 1.96 & 2598.0 & 24.5 & 0.185 & 2642.3 & 21.0 & 0.769 & 2664.1 & 29.2 & 0.554 & 2684.3 & 21.5 & 0.228 \\
\hline 2.33 & 2645.6 & 24.1 & 0.183 & 2678.8 & 19.1 & 0.691 & 2699.3 & 28.2 & 0.787 & 2716.7 & 20.0 & 0.254 \\
\hline 2.41 & 2651.3 & 24.1 & 0.192 & 2682.8 & 20.1 & 0.676 & 2703.2 & 29.5 & 0.829 & 2718.9 & 20.1 & 0.285 \\
\hline 2.54 & 2668.4 & 22.2 & 0.190 & 2696.8 & 22.8 & 0.672 & 2719.2 & 29.6 & 0.872 & 2732.0 & 19.7 & 0.206 \\
\hline \multicolumn{13}{|c|}{ calculations } \\
\hline $\begin{array}{l}\omega_{L} \\
{[\mathrm{eV}]}\end{array}$ & $\begin{array}{c}\omega_{1} \\
{\left[\mathrm{~cm}^{-1}\right]}\end{array}$ & $\begin{array}{c}\Gamma_{1} \\
{\left[\mathrm{~cm}^{-1}\right]}\end{array}$ & $\begin{array}{c}a_{1} \\
{[\operatorname{arb} . \text { u.] }}\end{array}$ & $\begin{array}{c}\omega_{2} \\
{\left[\mathrm{~cm}^{-1}\right]}\end{array}$ & $\begin{array}{c}\Gamma_{2} \\
{\left[\mathrm{~cm}^{-1}\right]}\end{array}$ & $\begin{array}{l}\quad a_{2} \\
{[\operatorname{arb} . \mathrm{u} .]}\end{array}$ & $\begin{array}{c}\omega_{3} \\
{\left[\mathrm{~cm}^{-1}\right]}\end{array}$ & $\begin{array}{c}\Gamma_{3} \\
{\left[\mathrm{~cm}^{-1}\right]}\end{array}$ & $\begin{array}{c}\quad a_{3} \\
\text { [arb. u.] }\end{array}$ & $\begin{array}{c}\omega_{4} \\
{\left[\mathrm{~cm}^{-1}\right]}\end{array}$ & $\begin{array}{c}\Gamma_{4} \\
{\left[\mathrm{~cm}^{-1}\right]}\end{array}$ & $\begin{array}{c}a_{4} \\
\text { [arb. u.] }\end{array}$ \\
\hline 1.96 & 2607.8 & 22.1 & 0.166 & 2655.8 & 24.4 & 0.934 & 2685.0 & 28.2 & 0.467 & 2696.8 & 15.8 & 0.294 \\
\hline 2.33 & 2649.1 & 23.9 & 0.182 & 2691.7 & 30.6 & 0.932 & 2725.0 & 25.6 & 0.764 & & & \\
\hline 2.41 & 2657.1 & 25.3 & 0.175 & 2699.0 & 32.6 & 0.922 & 2732.2 & 26.3 & 0.797 & & & \\
\hline 2.54 & 2668.4 & 24.3 & 0.149 & 2710.5 & 38.0 & 0.875 & 2744.1 & 28.0 & 0.810 & & & \\
\hline
\end{tabular}

energies. As already stated in the main part of our letter, the decomposition for laser energies up to $2.54 \mathrm{eV}$ follows that for $1.96 \mathrm{eV}$.

* fhz@physik.tu-berlin.de

[1] M. Lazzeri, C. Attaccalite, L. Wirtz, and F. Mauri, Phys. Rev. B 78, 081406 (2008).

[2] A. Grüneis, J. Serrano, A. Bosak, M. Lazzeri, S. L. Molodtsov, L. Wirtz, C. Attaccalite, M. Krisch, A. Rubio, F. Mauri, and T. Pichler, Phys. Rev. B 80, 085423 (2009).

[3] P. Venezuela, M. Lazzeri and F. Mauri, Phys. Rev. B 84, 035433 (2011).

[4] M. Calandra, G. Profeta, and F. Mauri, Phys. Rev. B 82, 165111 (2010).

[5] J. A. Yan, W. Y. Ruan, and M. Y. Chou, Phys. Rev. B 77, 125401 (2008).

[6] K. F. Mak, F. H. da Jornada, K. He, J. Deslippe, N. Petrone, J. Hone, J. Shan, S. G. Louie and T. F. Heinz, Phys. Rev. Lett. 112, $207401(2014)$.

[7] F. Kadi and E. Malic, Phys. Rev. B 89, 045419 (2014).

[8] D. M. Basko, Phys. Rev. B 78, 125418 (2008).

[9] D. L. Mafra, L. M. Malard, S. K. Doorn, H. Htoon, J. Nilsson, A. H. Castro Neto and M. A. Pimenta, Phys. Rev. B 80, 241414 (2009). 


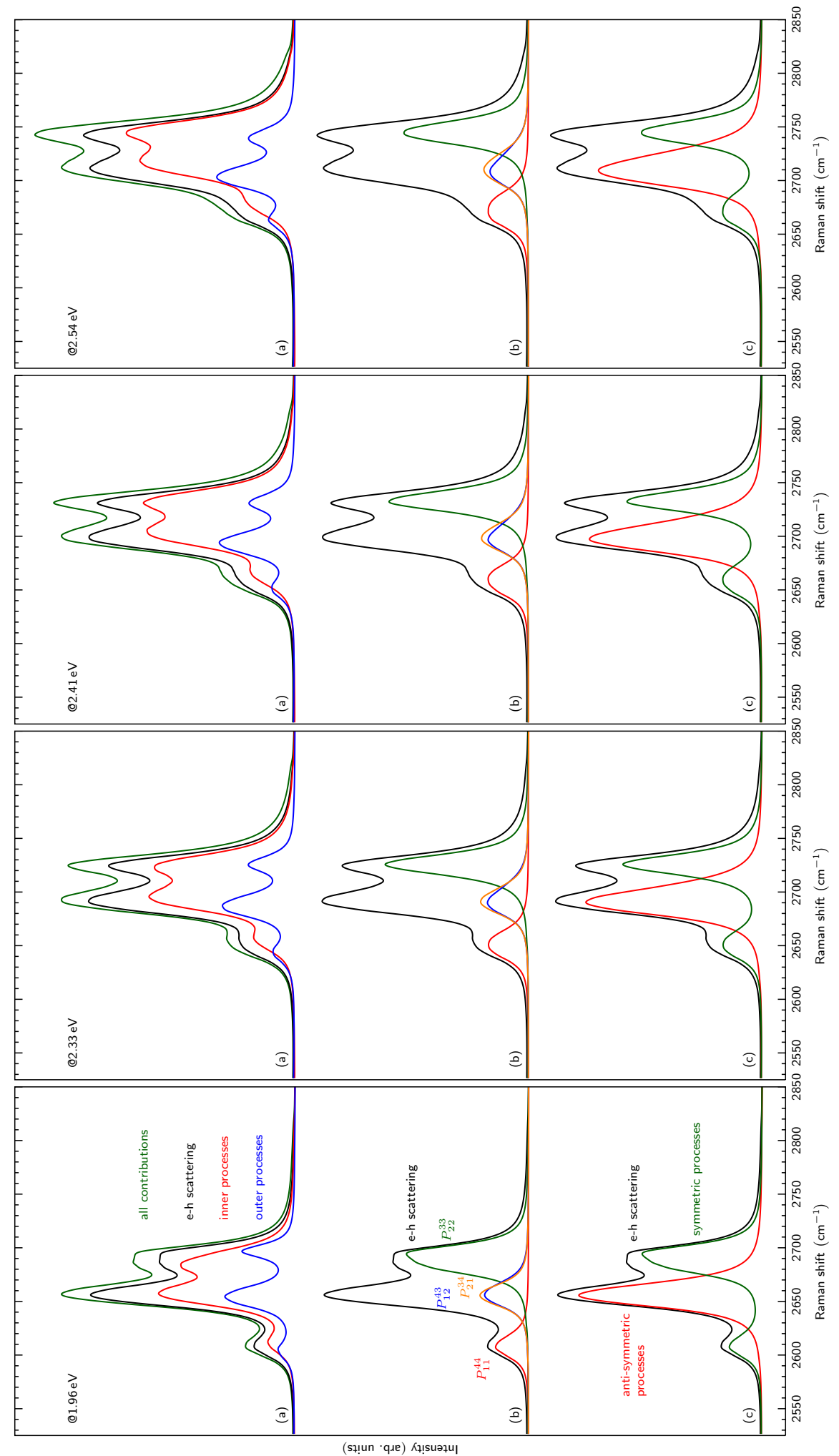

Figure 5. Decompostion of the calculated Raman spectra for four different laser excitation energies into (a) inner and outer contributions, as well as only e-h scattering, (b) the four different scattering processes (without interference between the single processes), and (c) symmetric and anti-symmetric scattering processes (including interference). 